
A. Handa
G. Abdo
H. Yilmaz
S. G. Wetzel
K. O. Lovblad
P. Bijlenga
N. De Tribolet
D. A. Rufenacht

\section{Double-lumen balloon microcatheter-assisted occlusion of cerebral vessels with coils: a technical note}

Received: 25 October 2003

Accepted: 8 March 2004

Published online: 5 June 2004

(C) Springer-Verlag 2004

\author{
A. Handa - G. Abdo · H. Yilmaz \\ S. G. Wetzel · K. O. Lovblad $(\bowtie)$ \\ D. A. Rufenacht \\ Division of Neuroradiology \\ of the Department of Radiology, \\ Geneva University Hospital, \\ Rue Micheli-du-Crest 24, \\ 1211 Geneva, Switzerland \\ E-mail: karl-olof.lovblad@hcuge.ch \\ Tel.: + 41-22-3727045 \\ Fax: + 41-22-3727072 \\ S. G. Wetzel \\ Division of Neuroradiology of the \\ Department of Radiology, Kantonsspital \\ Basel/University Clinics, Basel, \\ Switzerland \\ P. Bijlenga $\cdot$ N. De Tribolet \\ Department of Neurosurgery, \\ University Hospital, Geneva, Switzerland
}

\begin{abstract}
The purpose of this study was to describe a balloon-assisted double-lumen microcatheter technique to perform a controlled and tight coil packing of a vascular segment for vessel occlusion. This technique can be performed immediately after a test occlusion with the balloon kept in place and was, as illustrated in six cases, in our experience safe, straight forward to use and fast.
\end{abstract}

Keywords Double-lumen balloon microcatheter - Parent artery occlusion Endovascular technique · Test occlusion $\cdot$ Coil

\section{Introduction}

Endovascular parent artery occlusion may be used to treat a variety of vascular disease, such as aneurysms, pseudoaneurysms and arterio-venous fistulas. Vessel occlusion is also considered in some cases to facilitate tumor surgery. The current standard-of-procedure techniques request test occlusion prior to permanent vessel occlusion. To obtain a reliable result of the test occlusion, it is usually required to position a temporary balloon occlusion at the same level as the permanent occlusion is planned. Test occlusion is performed over a period of about $30 \mathrm{~min}$ or interrupted, if not tolerated.
During the occlusion test, currently evaluation includes angiographical visualization of satisfactory collateral supply and clinical neurological and neuropsyochological tests without signifcant changes, even under additional hemodynamic stress, i.e., a condition simulated with drug-induced lowering of the blood pressure after initial tolerance has been revealed. Additional tests may include semi-quantitative measurements of brain perfusion using EDC SPECT [1].

Formerly, as in other centers, non-detachable silicone balloons (Endeavour, 8501, BSC) for temporary and detachable latex balloons (Nycomed) for permanent parent artery vessel occlusion have been used most 
frequently $[1,2,3,4]$. With the advent of detachable platinum coils, and because of the lack of commercially available coaxially detachable balloon systems, there has been a trend to use coil occlusion to achieve permanent arterial occlusion [5]. With regard to technical aspects during coil placement, Graves et al. [6] reported the utility of temporary flow arrest by using a non-detachable balloon in order to reduce the risk of distal emboli and preclude difficulties in deploying coils in the arterial flow stream. We describe a modification of this technique. Thereby, a double-lumen balloon was used that allowed, after test occlusion, the obtaining of a tight packing of the vessel with detachable platinum alloy coils in the immediate vicinity of the balloon.

\section{Materials and methods}

Methods

Six patients underwent balloon test occlusion and subsequent temporary flow arrest using double-lumen balloon microcatheter-assisted and endovascular vessel occlusion with microcoils at our institution from March 2002 to April 2003. The patients included two males and four females, who ranged in age from to 28 to 71 years (mean 42.7 years). Three carotid arteries and three vertebral arteries were treated using this technique. Two patients had endovascular occlusion for unruptured internal carotid ophthalmic artery aneurysms, two for dissecting aneurysms of vertebral arteries, two for presurgical tumor resection of an invasive malignant tumor of the nerve sheaths at the cervical level and of a glomus jugular tumor (Table 1).

Balloon test occlusion was performed in each patient before the permanent occlusion. This procedure was carried out in awake patients under local anesthesia and light sedation. A bilateral femoral approach was established using a 6-7 F and a 4-5 F, respectively. After endovascular access was gained, the procedure was done in the following way (Fig. 1): (1) an occlusion test was performed using a microballoon catheter (Grapewine) inflated just proximal to the planned occlusion site. This was carried out like usual occlusion tests (see Introduction) and was tolerated without neurological deficit in all patients. (2) For definitive treatment, the balloon

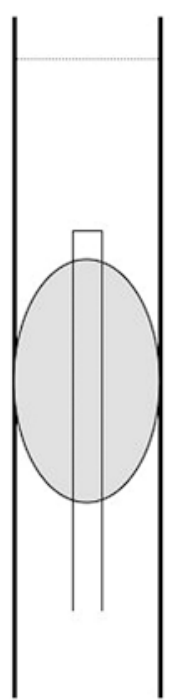

(1)

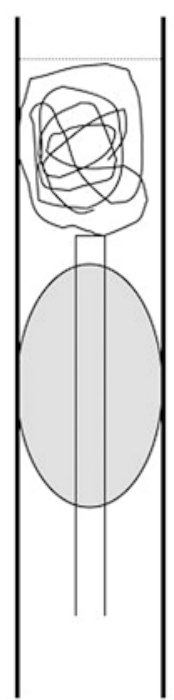

(2)

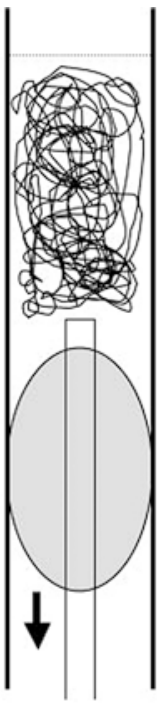

(3)

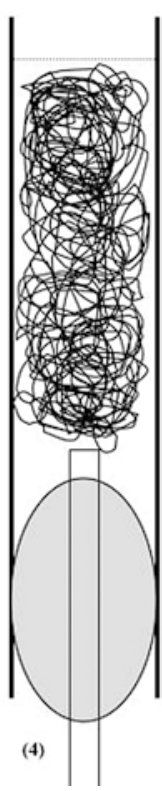

Fig. 1 Drawing of the double-lumen balloon catheter-assisted vessel occlusion technique. (1) Test occlusion: the balloon is inflated slightly proximal to the occlusion site. (2) First coils: spiral or spherical coils are deployed to form a stable basket ("anchor'). (3) Subsequent coils: now spiral, spherical as well as long J-shaped coils can be used to fill the space between the "anchor" and the balloon. Here, a slight backward movement of the balloon is observed. (4) Permanent occlusion

was kept inflated in place, and microcoils were introduced through the double-lumen balloon microcatheter to form a stable basket. For this purpose, we chose either a spiral or spherical coil of a slightly oversized dimension. (3-4) The creation of a coil distal basket was followed by placement of a series of microcoils until occlusion was thought to have been obtained. While microcoils were delivered, the balloon was kept in position, which allowed for packing the coils tightly in the space between the already introduced coils and the proximal continuously inflated balloon. In all cases, we could observe a stepwise backwards movement of the microballoon, whereas the distal coils stayed in the initial position, meaning that the pack of coils were in a stable position. The whole procedure was done under heparinization to keep the activated partial thromboplastin time of about twice that of the control.
Table 1 Summary of cases on double lumen balloon catheterassisted occlusion of cerebral vessels with coils

\begin{tabular}{llllll}
\hline Case & Age (years)/sex & Location & Diagnosis & Complications & Outcome \\
\hline 1 & $36 / F$ & Rt. IC & IC-oph An* & None & Good \\
2 & $49 / \mathrm{F}$ & Rt. VA & Dissecting An & None & Good \\
3 & $71 / \mathrm{F}$ & Lt. VA & Dissecting An & None & Good \\
4 & $32 / \mathrm{F}$ & Rt. VA & Invasion of tumor & None & Good \\
5 & $40 / \mathrm{F}$ & Rt. IC & IC-oph An* & None & Good \\
6 & $28 / \mathrm{F}$ & Rt. IC & Glomus jugular tumor & None & Good \\
\hline
\end{tabular}


Case examples:

In case 1, a 36-year-old woman with longstanding headache, but without an episode of subarachnoid hemorrhage, was found to have a right-sided internal carotid-ophtalmic artery aneurysm on MR angiography. Digital subtraction angiography showed a small carotid cave aneurysm and adjacent intradural ophthalmic aneurysm with a multilobular and irregular appearance of the right internal carotid artery (Fig. 2A, B). Due to technical difficulties encountered while trying to deliver stents in an in-vitro replica made of poly vinyl alcohol hydrogel replica, we decided to treat the patient's large carotid-ophthalmic aneurysm by endovascular occlusion of the internal carotid artery (ICA) at the level of the ophthalmic artery origin and

Fig. 2 A AP view, B lateral view. Cerebral digital subtraction angiography showed a small carotid cavernous aneurysm and adjacent intradural ophthalmic aneurysm with a multilobular and irregular appearance of the right internal carotid artery. C An occlusion test was performed using a balloon catheter inflated in close proximity to the aneurysm (segment $\mathrm{C} 4$ of the carotid siphon). For definitive treatment, the balloon was kept inflated in place, and a first spherical coil was introduced through the doublelumen balloon microcatheter to form a stable anchor in the artery at the level of the aneurysm neck. D While the J-coils were delivered, the balloon was kept in position, which allowed the coil to form a tight packing between the distal "coil-anchor" (the already introduced coils) and the proximal inflated balloon. E The final control showed a total occlusion extending proximally. Under anticoagulation using 4,000 IU of heparin, an occlusion test was performed using a 4-mm diameter Grapevine catheter-10 system (Medtronics Micro Interventional Systems, Sunnyvale, Calif.) inflated in close proximity to the aneurysm (segment C4 of the carotid siphon), which was tolerated without neurological deficit. For definitive treatment, the balloon was kept inflated in place, and a first spherical coil (Micrus coil: MICRUS CORPORATION, Sunnyvale, Calif.) was introduced through the double-lumen balloon microcatheter to form a stable anchor in the artery at the level of the aneurysm neck (Fig. 2C). This was followed by placement of 11 mechanically detachable coils (DCS-11, COOK) for a total length of $79 \mathrm{~cm}$ of coils. The initial introduction of spiral coils was followed by delivery of long $\mathrm{J}$-shaped coils. While the J-coils were delivered, the balloon was kept in the position, which allowed the coil to form tight packing between the distal "coilanchor" (the already introduced coils) and the proximal inflated balloon (Fig. 2D). The final control showed a total occlusion (Fig. 2E). No neurological symptoms occurred during or after coil embolization, and the follow-up at 1 year showed disappearance of the aneurysm, a permanent occlusion of the right internal carotid artery and normal appearance of the brain.

In case 6, a 28-year-old woman with a large right glomus jugular tumor showed encasement of the

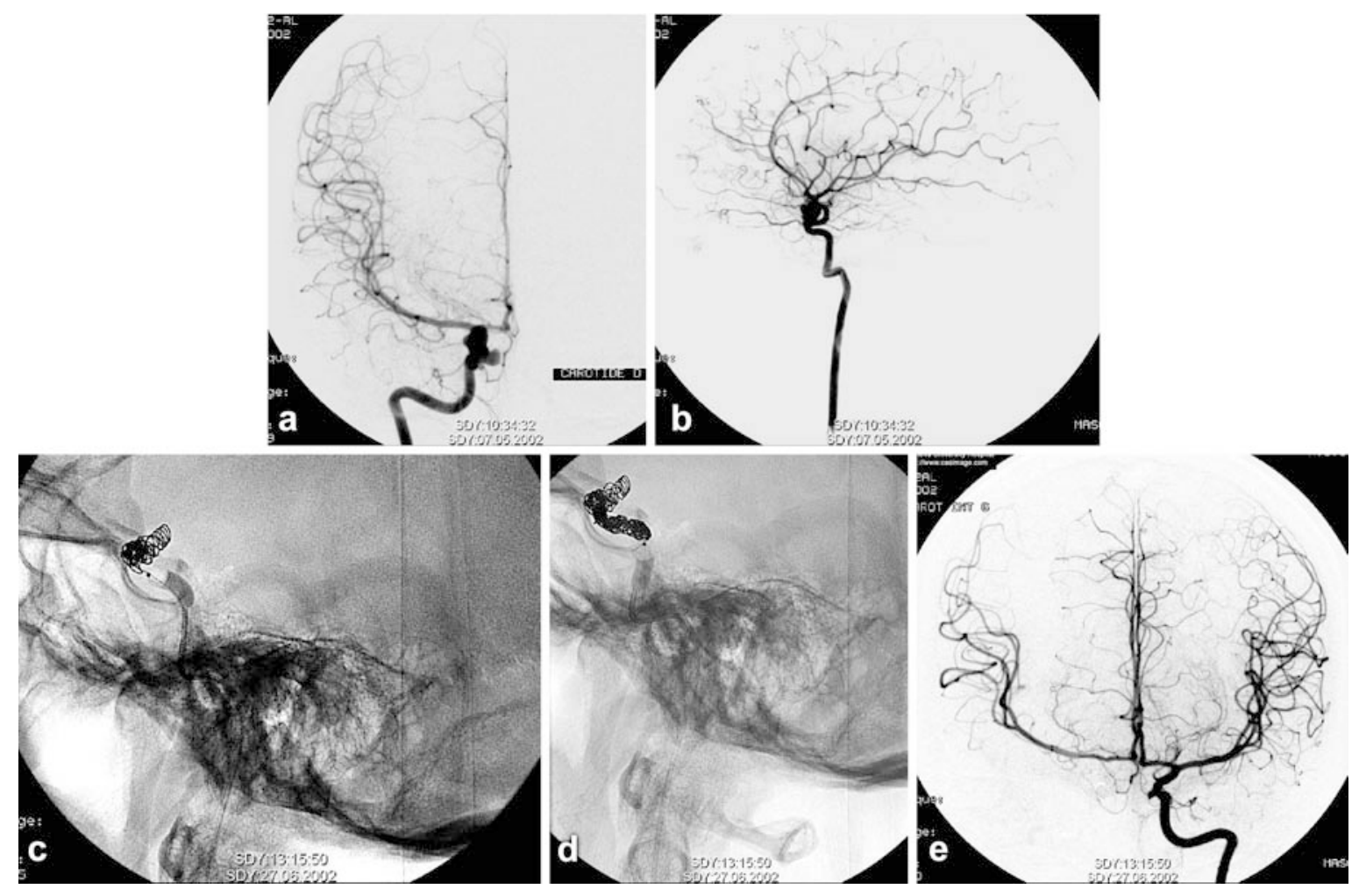


Fig. 3 A Lateral view: a large right glomus jugulare tumor showed encasement of the petrous segment of the internal carotid artery. B Lateral view: on the balloon test occlusion, the balloon catheter was inflated at the $\mathrm{C} 4$ segment of the carotid siphon. C Lateral view: the permanent vessel occlusion was carried out while proximal flow arrest was maintained. D Lateral view: a second proximal occlusion of the cervical ICA was performed to achieve trapping. E Lateral view: the final control showed a total occlusion of the ICA

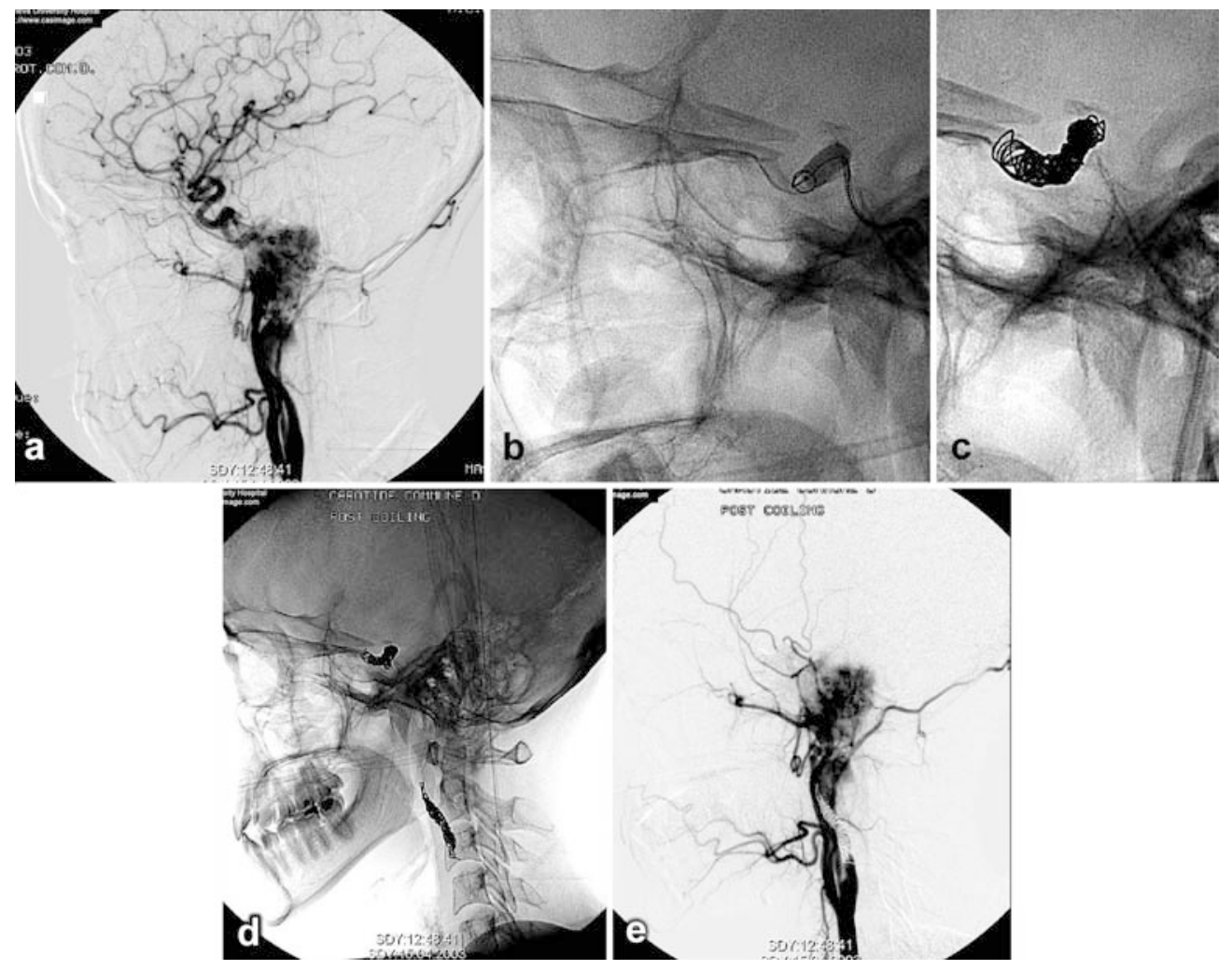

petrous segment of the internal carotid artery (Fig. 3A). Among the presurgical preparations involving devascularization of the tumor, the occlusion of the right ICA was requested. Since tumor resection including resection of the petrous segment of the ICA was planned, we performed balloon test occlusion at the $\mathrm{C} 4$ segment of the carotid siphon, with the intention to coil the $\mathrm{C} 3$ and the $\mathrm{C} 4$ segments of the ICA. A 4-mm diameter Grapevine catheter-10 (Medtronics Micro Interventional systems, Sunnyvale, Calif.) was inflated, and clinical testing revealed no neurological and neuropsychological deficits. There was a slight asymmetry at the parenchymography study and back-pressure measurements revealed a drop of the mean pressure from 80 to $48 \mathrm{mmHg}$. The permanent vessel occlusion was carried out while proximal flow arrest was maintained in a manner similar to that described in case 1 (Fig. 3B, C). Since a segmental resection of the petrous portion of the ICA was planned, we avoided introducing coils in this portion, and a second proximal occlusion of the cervical ICA was performed to achieve trapping. Again, a similar coil introduction technique was used (Fig. 3D). For the distal occlusion, a total of 14 coils for a total length of $117.6 \mathrm{~cm}$ of coils was used, and for the proximal occlusion these numbers were 7 coils for a total of $85.6 \mathrm{~cm}$. A combination of 0.010 and 0.018 coil systems were used.
The final control showed a total occlusion of the ICA (Fig. 3E). This was followed by other presurgial endovascular and direct puncture devascularization procedures. The whole procedure was done under heparinization to keep the activated partial thromboplastin time of about twice that of the control, and anticoagulation was maintained at preventive levels over night. No neurological symptoms occurred during or after coil embolization, and surgical removal was facilitated, satisfying the surgeon's expectations.

\section{Results}

All six parent arteries were occluded successfully, and occlusion length could be limited to the intended extension. All lesions were successfully treated, resulting in no complications, no neurological deficits and good outcomes. Permanent vessel occlusion was performed using 10 to 29 coils (either GDC, DCS or Micrus) with a total length of 79 to $185 \mathrm{~cm}$.

The above-described technique allowed the performance of a controlled and tight coil packing of the vascular segment intended to be occluded. This technique was performed immediately after the test occlusion with the balloon kept in place and was, as illustrated in the six cases, in our experience safe, straight forward to use and fast. 


\section{Discussion}

Regarding the procedural aspects of parent vessel occlusion with microcoils, in two case series different techniques have been described. Graves et al. [6] passed a microcatheter through the lumen of a still-inflated test occlusion balloon to the level selected for permanent occlusion to deploy the coils. Their rationale for applying a proximal flow arrest was two-fold: first, flow arrest has been shown to reduce the risk of distal emboli during coil deployment in a canine model [7]. Second, the authors found flow arrest to reduce the risk of coil migration and to allow for precise placement of the coils. In contrast, Barr et al. [8] subsequently reported that, according to their results, flow arrest for coil placement was unnecessary if intravenous heparin and heparinized catheter flush solutions were used. They remarked that in the canine model cited above, anticoagulation had not been employed. Choosing coils that were 33 to $50 \%$ larger than the diameter of the artery to be occluded as the first coils placed, they encountered no inadvertent distal coil migration.

We used a slightly modified technique compared to that described by Graves et al. [8]. It differed from their technique in two aspects. First, the double-lumen balloon allowed for test occlusion close to the intended occlusion site. Second, the function of the double-lumen balloon was to pass the coils directly to the site elected for permanent artery occlusion without the need of bringing in a separate microcatheter or balloon deflation after the successful testing. Third, the microballoon helped the coil deployment, similar to techniques applied when performing "remodeling techniques" [10] during aneurysm coiling. After initial introduction of slightly oversized spherical or spiral coils, which form an anchor, it was possible to keep the vessel occlusion over a short segment involving a minimum of about $2.5 \mathrm{~cm}$ for the size of a $5 \mathrm{~mm}$ lumen ICA. The balloon assistance further allowed for use of long J-shaped coils (DCS, COOK), as the balloon served as the proximal limit preventing the proximal extension of unshaped coils. In our experience, use of such $\mathrm{J}$-coils may reduce significantly the number of coils required to obtain a reliable vessel occlusion. Furthermore, mechanically detachable coils exhibit features that make them advantageous for parent vessel occlusion [8] because of its rapid and reliable detachment time when compared to other detachment systems. Detachment of mechanically detachable coils was in our cases no more than $25 \mathrm{~s}$ per coil. When unavailable, instead of using a doublelumen balloon microcatheter, one could use two microcatheter systems that are used independently for temporally occluding and for delivery to the coils, respectively.

The currently described detachable balloon technique for vessel occlusion has some advantages, for example, it is easier to perform test occlusion and detach the balloon just after the test is finished, and it is cheaper than coiling. However, there is the risk of a premature detachment, perhaps slightly reducing patient safety. Furthermore, there is a lack of commercial availability of coaxial detachable balloon systems.

\section{Conclusions}

Following a test occlusion, endovascular occlusion of the parent artery can be accomplished in a fast, accurate and effective way with coils assisted by a double-lumen balloon kept in place inflated.

\section{References}

1. Serbinenko FA (1974) Balloon catheterization and occlusion of major cerebral vessels. J Neurosurg 41:125-145

2. Larson JJ, Tew JM Tomsick TA, van Loveren HR (1995) Treatment of aneurysms of the internal carotid artery by intravascular balloon occlusion: long-term follow-up of 58 patients. Neurosurgery 36:26-30
3. Lewis AI, Tomsick TA, Tew JM (1995) Management of 100 consecutive direct carotid-cavernous fistulas: results of treatment with detachable balloons. Neurosurgery 36:239-244

4. Fox AJ, Vinuela F, Pelz DM, Peerless SJ, Ferguson GG, Drake CG, Debrun G (1987) Use of detachable balloons for proximal artery occlusion in the treatment of unclippable cerebral aneurysms. J Neurosurg 66:40-46

5. Braun IF, Hoffman JC Jr, Casarella WJ, Davis PC (1985) Use of coils for transcatheter carotid occlusion. Am J Neuroradiol 6:953-956
6. Graves VB, Perl J 2nd, Strother CM, Wallace RC, Kesava PP, Masaryk TJ (1997) Endovascular occlusion of the carotid or vertebral artery with temporary proximal flow arrest and microcoils: clinical results. Am J Neuroradiol 18:1201-1206

7. Hughes SR, Graves VB, Kesava PP, Rappe AH (1996) The effect of flow arrest on distal embolic events during arterial occlusion with detachable coils: a canine study. Am J Neuroradiol 17:685-691 
8. Barr JD, Lemley TJ (1999) Endovascular arterial occlusion accomplished using microcoils deployed with and without proximal flow arrest: results in 19 patients. Am J Neuroradiol 20:14521456
9. Cloft HJ, Kallmes DF, Jensen ME, Lanzino G, Dion JE (1999) Endovascular treatment of ruptured, peripheral cerebral aneurysms: parent artery occlusion with short Guglielmi detachable coils. Am J Neuroradiol 20:308310
10. Murphy KJ, Houdart E, Szopinski KT, Levrier O, Guimaraens L, Kuhne D, Solymosi L, Bartholdy NJ, Rufenacht DA (2001) Mechanical detachable platinum coil: report of the European phase II clinical trial in 60 patients. Radiology 219:541-544 